\title{
AVALIAÇÃO DOS TRATAMENTOS ELETROQUÍMICO E FOTOELETROQUÍMICO NA DEGRADAÇÃo DE
} CORANTES TÊXTEIS

\author{
Marciana Catanho, Geoffroy Roger Pointer Malpass e Artur de Jesus Motheo*
}

Instituto de Química de São Carlos, Universidade de São Paulo, CP 780, 13560-970 São Carlos - SP, Brasil

Recebido em 22/8/05; aceito em 13/1/06; publicado na web em 14/6/06

\begin{abstract}
EVALUATION OF ELECTROCHEMICAL AND PHOTOELECTROCHEMICAL METHODS FOR THE DEGRADATION OF THREE TEXTILE DYES. This paper presents the study of the oxidation of three textile dyes (Remazol black B, Remazol Brilliant Orange 3R and Remazol Golden Yellow RNL) using electrochemical and photoelectrochemical methods. In both methods, electrolysis experiments were performed at a current density of $50 \mathrm{~mA} \mathrm{~cm}{ }^{-2}$ in an aqueous solution of each dye (30 $\left.\mathrm{mg} \mathrm{L}^{-1}\right)$, using a photoelectrochemical flow-cell. For all the dyes studied, the photoelectrochemical method was demonstrated to be more efficient than the electrochemical one. Photoelectrochemical oxidation resulted in complete decoloration after 90 min of electrolysis and total organic carbon (TOC) removal reached up to 36\%. It was observed that the dyes presenting chromophores at higher wavelengths are removed the quickest, which indicates that photosensitised (photoassisted) oxidation occurs. The level of color was reduced to levels below the standards presented in the literature, which indicates the viability of the photoelectrochemical process as part of the treatment of textile effluents.
\end{abstract}

Keywords: azo dye; reactive dye; DSA.

\section{INTRODUÇÃO}

Os resíduos de indústrias têxteis possuem como característica uma intensa coloração a qual, em ambientes aquáticos, pode causar uma interferência nos processos de fotossíntese. Além disso, certas classes de corantes, assim como seus subprodutos, podem ser carcinogênicos e/ou mutagênicos ${ }^{1}$. Estudos ${ }^{2}$ indicam que a poluição colorida de cursos d'água começa a ser observável a concentrações acima de $1 \mathrm{mg} \mathrm{L}^{-1}$. Além disso, resíduos da indústria têxtil podem conter metais pesados em níveis acima dos permitidos pelas leis ambientais ${ }^{3}$.

A produção mundial de corantes e pigmentos é estimada entre 750.000 e 800.000 t/ano ${ }^{2,3}$ sendo que cerca de 26.500 t/ano são consumidas no Brasil ${ }^{1}$. Desta quantidade, aproximadamente $12 \%$ dos corantes orgânicos são perdidos durante as etapas de produção e processamento $^{1}$. Esta perda é relativamente pequena comparada com outros poluentes, como pesticidas, solventes e detergentes ${ }^{1}$; porém, é importante enfatizar que a aplicação destes materiais geralmente envolve diluição, gerando um grande volume de efluente líquido.

A classificação dos corantes pode ser pelo tipo de fibra, tais como corantes para nylon, algodão, poliéster, etc.; pelos métodos de aplicação no substrato, ou seja, pela maneira que eles são fixados à fibra têxtil (corantes diretos, reativos, à cuba, etc.), e de acordo com a sua estrutura química, como por ex.: azo, antraquinona, indigóides, etc. $)^{47}$. Cerca de $60 \%$ dos corantes utilizados em indústrias têxteis são corantes azos, que se caracterizam pelo grupo $-\mathrm{N}=\mathrm{N}$ - ligados a sistemas aromáticos ${ }^{1}$, sendo que a função azo inclui os principais tipos de corantes reativos. Os corantes reativos contêm um grupo eletrofílico (reativo) capaz de formar ligação covalente com grupos hidroxila das fibras celulósicas, com grupos amino, hidroxila e tióis das fibras protéicas e também com grupos amino das poliamidas. Devido às grandes quantidades de corantes que não são fixados às fibras e são liberados no processamento têxtil, é importante que seja estudada a degradação destes corantes em condições (de pH,

*e-mail: artur@iqsc.usp.br condutividade, etc.) semelhantes àquelas dos efluentes que são descartados. Ou seja, são necessários estudos laboratoriais reproduzindo as condições encontradas nas fábricas, de forma que a metodologia possa vir a ser aplicada.

Vários processos são apresentados na literatura para a descoloração de efluentes têxteis, tais como biológico ${ }^{8-12}$, sorção utilizando o cucurbituril ${ }^{13,14}$, precipitação química, oxidação química, adsorção e suas combinações ${ }^{15}$ e fotocatálise ${ }^{16,17}$.

\section{Oxidação fotocatalítica}

Desde meados dos anos 80 existe um grande interesse na utilização de materiais semicondutores para mineralização fotocatalítica de poluentes orgânicos ${ }^{16,17}$. Dentre os estudos com semicondutores, o mais investigado é o com dióxido de titânio $\left(\mathrm{TiO}_{2}\right)$ devido às seguintes características: alta reatividade fotoquímica, baixo custo, estabilidade em sistemas aquosos e baixa toxidade ambiental ${ }^{18-24}$. Konstantinou e Albanis ${ }^{25}$ revisaram a degradação fotocatalítica de corantes azo contendo diferentes funcionalidades, utilizando $\mathrm{TiO}_{2}$ em solução aquosa e sob radiação ultravioleta. Apresentaram evidências de que o mecanismo de fotodegradação é dependente de diferentes parâmetros: tipo de radiação utilizada, $\mathrm{pH}$, concentração de catalisador, concentração de substrato e presença de aceptores de elétrons, tais como peróxido de hidrogênio e persulfato de amônia, além de oxigênio molecular.

Quando um semicondutor é irradiado com radiação ultravioleta, a uma energia maior que sua energia de "bandgap", são gerados elétrons $\left(\mathrm{e}_{\mathrm{CB}}{ }^{-}\right)$na banda de condução e lacunas $\left(\mathrm{h}_{\mathrm{VB}}{ }^{+}\right)$na banda de valência. Os elétrons fotogerados podem reagir tanto com o corante quanto com elétrons aceptores, por ex., moléculas de oxigênio adsorvidas na superfície do semicondutor ou dissolvidas na água, gerando um superóxido $\mathrm{O}_{2}{ }^{-}$. As lacunas fotogeradas podem oxidar a molécula orgânica formando $\mathrm{R}^{+}$, ou reagir com $\mathrm{OH}^{-}$ou $\mathrm{H}_{2} \mathrm{O}$ oxidando estas espécies a $\mathrm{OH}^{*}$. Outras espécies altamente oxidantes (por ex., radicais de água oxigenada) são responsáveis pela fotodecomposição heterogênea com semicon- 
dutores nos substratos orgânicos, tais como corantes. As reações relevantes que ocorrem na superfície do semicondutor que levam à degradação de corantes são $^{25}$ :

$\mathrm{TiO}_{2}+\mathrm{hv}(\mathrm{UV}) \rightarrow \mathrm{TiO}_{2}\left(\mathrm{e}_{\mathrm{CB}}{ }^{-}+\mathrm{h}_{\mathrm{VB}}{ }^{+}\right)$

$\mathrm{TiO}_{2}\left(\mathrm{~h}_{\mathrm{vB}}{ }^{+}\right)+\mathrm{H}_{2} \mathrm{O} \rightarrow \mathrm{TiO}_{2}+\mathrm{H}^{+}+\mathrm{OH}^{\cdot}$

$\mathrm{TiO}_{2}\left(\mathrm{~h}_{\mathrm{vB}}^{+}\right)+\mathrm{OH}^{-} \rightarrow \mathrm{TiO}_{2}+\mathrm{OH}^{\cdot}$

$\mathrm{TiO}_{2}\left(\mathrm{e}_{\mathrm{CB}}{ }^{-}\right)+\mathrm{O}_{2} \rightarrow \mathrm{TiO}_{2}+\mathrm{O}_{2} \cdot$

$\mathrm{O}_{2}^{--}+\mathrm{H}^{+} \rightarrow \mathrm{HO}_{2}^{\cdot}$

Corante $+\mathrm{OH}^{\cdot} \stackrel{2}{\rightarrow}$ produtos de degradação

Corante $+\mathrm{h}_{\mathrm{VB}}{ }^{+} \rightarrow$ produtos de oxidação

Corante $+\mathrm{e}_{\mathrm{CB}} \rightarrow$ produtos de redução

$\mathrm{O}$ radical $\mathrm{OH}^{*}$ resultante é um agente oxidante muito forte e pode oxidar a maioria dos corantes. Deve ser notado que a oxidação fotosensibilizada pode ocorrer, a qual se constitui da excitação por luz visível (não UV) do corante e sua subseqüente interação com o semicondutor ${ }^{26,27}$ :

Corante + hv(VIS $) \rightarrow$ Corante*

Corante* $+\mathrm{TiO}_{2} \rightarrow$ Corante ${ }^{+\bullet}+\mathrm{TiO}_{2}(\mathrm{e})$

$\mathrm{TiO}_{2}(\mathrm{e})+\mathrm{O}_{2} \rightarrow \mathrm{TiO}_{2}+\mathrm{O}_{2}^{-*}$

$\mathrm{O}_{2}^{-\cdot}+\mathrm{TiO}_{2}(\mathrm{e})+2 \mathrm{H}^{+} \rightarrow \mathrm{H}_{2} \mathrm{O}_{2}$

$\mathrm{H}_{2} \mathrm{O}_{2}+\mathrm{TiO}_{2}(\mathrm{e}) \rightarrow{ }^{\cdot} \mathrm{OH}+\mathrm{OH}^{-}$

Corante $^{+\bullet}+\mathrm{O}_{2}\left(\mathrm{O}_{2}^{-\bullet}\right.$ ou $\left.\mathrm{OH}^{*}\right) \rightarrow$ peroxilados ou intermediários

hidroxilados $\rightarrow$ produtos degradados ou mineralizados

Quando é utilizada luz solar ou uma fonte de luz artificial, é possível que ambos os mecanismos (fotooxidação e fotossensibilização) ocorram, tornando difícil distinguir se o mecanismo de oxidação fotocatalítica é superior ao de oxidação fotossensibilizada.

Um fator que diminui a eficiência do processo fotoquímico é a recombinação do elétron com $\mathrm{h}^{+}$(o inverso da reação 1 ), antes do mesmo interagir com o corante ${ }^{28}$. Uma forma de evitar esta recombinação é utilizar a irradiação UV com aplicação simultânea de um potencial externo anódico sobre um eletrodo semicondutor, forçando assim a separação das espécies carregadas ${ }^{28}$. A esta combinação dos métodos eletroquímico e fotoquímico é dada a denominação de fotoeletrocatálise.

A área de fotoeletrocatálise é relativamente nova mas mostra um crescente número de trabalhos a cada ano. Originalmente, o avanço da área foi limitado pelo fato de que a maioria dos fotocatalisadores existia somente na forma de pó em suspensão, o que necessitava de separação do meio reacional pós-tratamento. Desta forma, o desenvolvimento de técnicas para imobilizar materiais semicondutores em suportes foi crucial no desenvolvimento desta área ${ }^{29}$. A oxidação fotoeletrocatalítica tem se mostrado eficaz no tratamento de diversas substâncias orgânicas que são poluentes ou danosas à saúde, como 4-clorofenol ${ }^{30-33}$, ácido fórmico $^{34-36}$ e até organismos patogênicos ${ }^{37}$. Destaca-se também o tratamento dos corantes naftol azul ${ }^{38}$, remazol brilhante laranja $3 \mathrm{R}^{39} \mathrm{e}$ naftol azul preto (sobre $\left.\mathrm{WO}_{3}\right)^{40}$, entre outros.

Dentre os possíveis materiais para uso na fotoeletrocatálise, destacam-se os chamados ânodos dimensionalmente estáveis (ADE), os quais se constituem de materiais semicondutores, imobilizados em um substrato inerte (e.g. Ti). Os ADEs tiveram destaque na década de 60 , substituindo os eletrodos de mercúrio na indústria cloro-alcali ${ }^{41}$. Entretanto, nos últimos 10 anos os ADEs também têm sido empregados em sistemas eletroquímicos para a degradação de substâncias orgânicas ${ }^{42-44}$, sendo que mais recentemente foi observado que os ADEs podem ser usados como fotocatalisadores $^{45-48}$. O uso de ADEs em sistemas fotoeletrocatalíticos tem sido feito para estudo da degradação de uma variedade de substâncias orgânicas, como, por ex., de efluentes da indústria de celulose, indústria têxtil e aterros sanitários ${ }^{46}$, do corante remazol vermelho $^{48}$ e de ácido húmico ${ }^{49}$.

Devido ao fato que o grande uso de corantes azo leva ao descarte descontrolado de efluentes coloridos, este trabalho apresenta o estudo da oxidação eletroquímica e fotoeletroquímica de três corantes azo: preto remazol $\mathrm{B}$, alaranjado remazol 3R e amarelo ouro remazol RNL (Esquema 1).
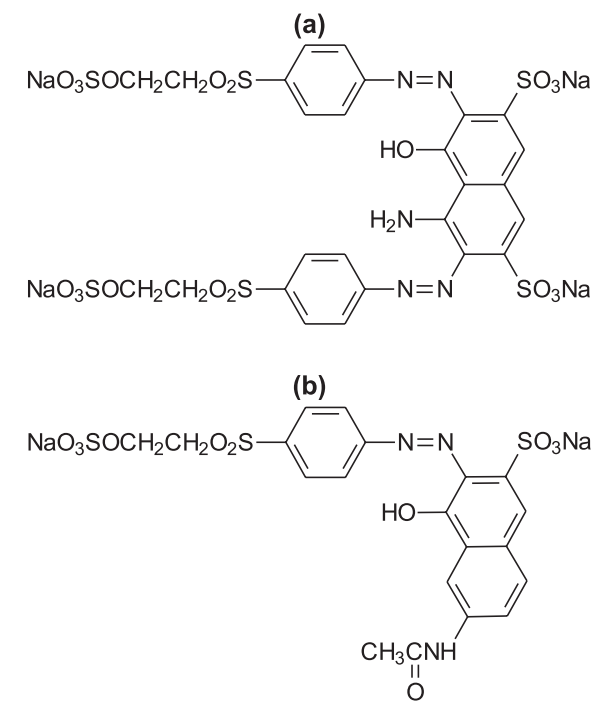

(c)

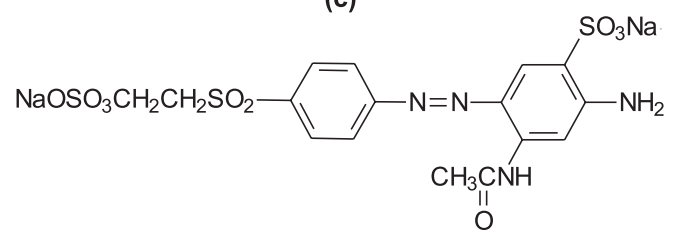

Esquema 1. Estruturas dos corantes: (a) preto remazol B; (b) alaranjado remazol $3 R$ e (c) amarelo ouro remazol $R N L$

Esses três corantes também são da mesma classe de classificação quanto à maneira que são fixados na fibra têxtil (tinturaria), sendo chamados de corantes reativos. São solúveis em água e reagem com algumas fibras têxteis, formando uma ligação do tipo covalente. É a mais importante classe de corantes utilizada hoje em dia, e pode ser usado em fibras celulósicas, em seda ou lã. São corantes que contêm um grupo eletrofílico (reativo) capaz de formar ligação covalente com grupos hidroxila das fibras celulósicas, com grupos amino, hidroxila e tióis das fibras protéicas e, também, com grupos amino das poliamidas. Os principais tipos de corantes reativos contêm a função azo e antraquinona como grupos cromóforos e os grupos clorotriazinila e sulfatoetilsulfonila como grupos reativos. A linha remazol da Hoechst, origem dos corantes utilizados, é composta por corantes reativos que contêm em sua estrutura um grupo sulfato-etilsulfônico $\left(\mathrm{SO}_{2} \mathrm{CH}_{2} \mathrm{CH}_{2}-\mathrm{OSO}_{3} \mathrm{H}\right)$, que é hidrolizado para vinil sulfônico $\left(\mathrm{SO}_{2} \mathrm{CH}=\mathrm{CH}_{2}\right)$ e forma uma ligação covalente forte com uma amina livre ou outro grupo substituinte na fibra, que é o caso dos três corantes escolhidos neste trabalho. Ao serem degradados estes corantes provavelmente produzirão produtos semelhantes, pois apresentam grupos semelhantes na sua estrutura química, lembrando que todos os corantes remazóis são fabricados com a mesma matéria-prima o PBE (para-base-éster), de fórmula molecular: 


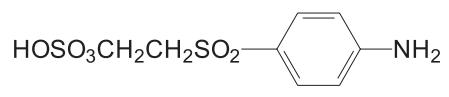

Esquema 2. Estrutura do para-base-éster (éster para amino fenil $\beta$ hidróxi etil sulfato)

\section{PARTE EXPERIMENTAL}

\section{Reagentes}

Foram utilizados três corantes têxteis, sem purificação prévia: preto remazol B (C.I. preto reativo 5), alaranjado remazol 3R (C. I. alaranjado reativo 16) e amarelo ouro remazol RNL (alaranjado reativo 107). As soluções aquosas de sulfato de sódio $\left(0,02 \mathrm{~mol} \mathrm{~L}^{-1}\right.$, Merck p.a.), com os corantes (30 $\left.\mathrm{mg} \mathrm{L}^{-1}\right)$, foram preparadas utilizando-se água ultrapura (Mili-Q, Milipore, resistência nominal $18 \mathrm{~m} \Omega$ ).

\section{Eletrodos e reator}

O eletrodo de trabalho (ET) foi um ADE de composição nominal $\mathrm{Ti} / \mathrm{Ru}_{0,3} \mathrm{Ti}_{0,7} \mathrm{O}_{2}$, obtido comercialmente (De Nora, Brasil), com área exposta à solução de $12 \mathrm{~cm}^{-2}$. $\mathrm{O}$ contra-eletrodo (CE) foi uma tela de titânio, de forma a possibilitar a irradiação da superfície do ânodo, através de uma janela de quartzo. Como eletrodo de referência (ER) foi utilizado um eletrodo de óxido mercuroso $(\mathrm{Hg} /$ $\mathrm{HgO})$ confeccionado no laboratório.

A irradiação do ânodo foi feita com o filamento de uma lâmpada de vapor de mercúrio de $250 \mathrm{~W}$ (Osram). O espectro da lâmpada de vapor de mercúrio foi medido, a fim de verificar a faixa de emissão de radiação correspondente à lâmpada. Pelo espectro da lâmpada foi observado que além do comprimento de onda do ultravioleta $(200$ - $400 \mathrm{~nm})$ essa também emitiu radiação na região do visível (400 - $750 \mathrm{~nm})$.

Para possibilitar o monitoramento do potencial do eletrodo de trabalho (ânodo) foi utilizada uma membrana de nafion (M), perfurada na região central, cuja terminação externa era inserida em solução eletrolítica em contato com o ER. De uma forma pouco ortodoxa, a membrana faz as vezes de um capilar de Luggin. O reator e a lâmpada ficaram dentro de uma caixa de madeira que foi acoplada a um exaustor, de forma a controlar a temperatura, e um termômetro para monitorar a temperatura no interior da caixa. Para circulação da solução foi utilizada uma bomba peristáltica com vazão de $18 \mathrm{~L} \mathrm{~h}^{-1}$.

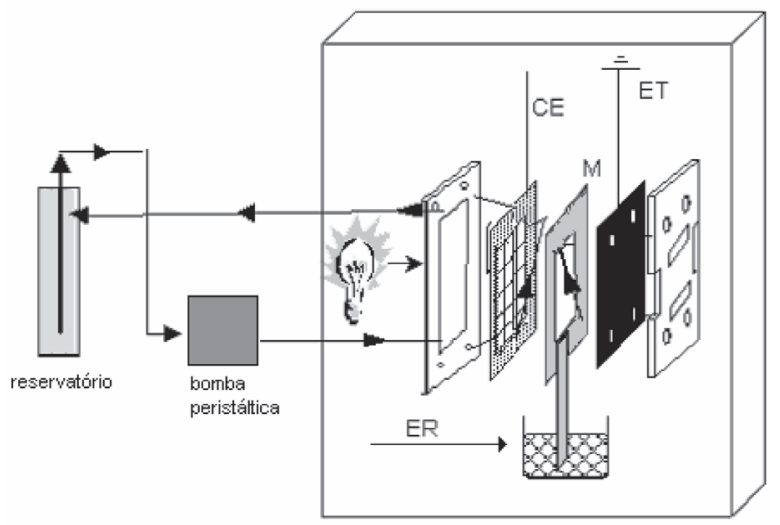

Figura 1. Esquema do reator fotoeletroquímico: CE: contra-eletrodo, ET: eletrodo de trabalho ( $\mathrm{Ti} / \mathrm{R} \mathrm{u}_{0,3} \mathrm{Ti}_{0,7} \mathrm{O}_{2}$ comercial); $\mathrm{M}$ : membrana de nafion; ER: local de inserção do eletrodo de referência

\section{Análises}

As eletrólises foram acompanhadas por espectroscopia Ultravioleta-visível (Varian Cary 2315) em uma faixa de comprimento de onda de 800 a $200 \mathrm{~nm}$ e as determinações de carbono orgânico total (COT) realizadas em um aparelho modelo TOC $\mathrm{V}_{\mathrm{CPH}}$ Total Organic Analyser (Shimadzu).

\section{Metodologias}

As eletrólises realizadas foram de $3 \mathrm{~h}$, a densidade de corrente de $50 \mathrm{~mA} \mathrm{~cm}{ }^{-2}$. A degradação fotocatalítica assistida eletroquimicamente (fotoeletroquímica) foi realizada acoplando a corrente elétrica simultaneamente com a irradiação de luz na superfície do eletrodo. Na degradação eletroquímica, a lâmpada foi apagada e somente a corrente foi aplicada.

\section{RESULTADOS E DISCUSSÃO}

\section{Análises de Voltametria Cíclica (VC)}

Os voltamogramas cíclicos do eletrodo $\mathrm{Ti} / \mathrm{Ru}_{0,3} \mathrm{Ti}_{0,7} \mathrm{O}_{2}$, na presença e ausência de irradiação ultravioleta, são apresentados na Figura 2, sendo que a Figura 2a corresponde ao perfil voltamétrico do eletrodo em 0,02 mol L-1 $\mathrm{Na}_{2} \mathrm{SO}_{4}$, sem adição do corante. Pode ser observado um aumento expressivo da área do voltamograma na região de 0 a $0,8 \mathrm{~V} v s$. EOM quando o eletrodo é irradiado. Adicionalmente, ocorre um deslocamento do começo da reação do desprendimento de oxigênio (RDO) para valores de potencial menos positivos. De acordo com Comninellis ${ }^{42}$, uma das condições para que a eletro-oxidação de espécies orgânicas ocorra em eletrodos de óxidos é que a RDO ocorra simultaneamente. Isto é devido ao fato que as espécies intermediárias da formação de $\mathrm{O}_{2}$ são responsáveis pela oxidação de substâncias orgânicas. Desta forma, o deslocamento do início da RDO que ocorre na presença de irradiação resultará em uma diminuição da energia necessária para oxidar a espécie orgânica.

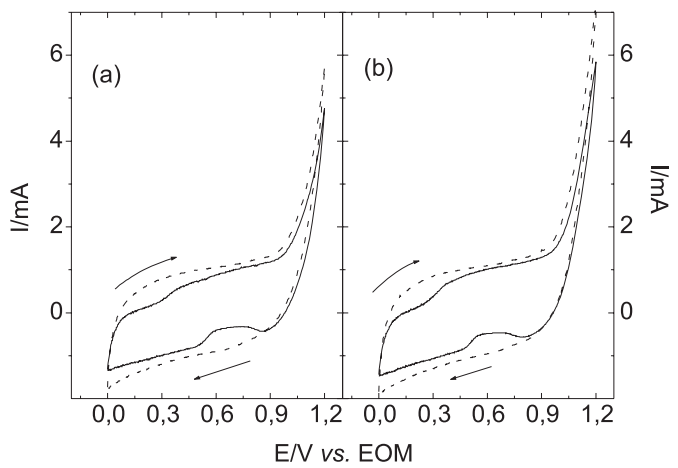

Figura 2. Voltamogramas cíclicos de $\mathrm{Ti} / \mathrm{Ru}_{0,3} \mathrm{Ti}_{0,7} \mathrm{O}_{2}$ em soluções de $\mathrm{Na}_{2} \mathrm{SO}_{4}$ $\left(0,02 \mathrm{~mol} \mathrm{~L}^{-1}\right)$ na ausência (a) e na presença (b) de corante (preto remazol B). (-) Sem e (--) com irradiação UV-vis. $v=20 \mathrm{mV} \mathrm{s}^{-1}$

A Figura $2 b$ apresenta o perfil voltamétrico na presença do corante PR (35 mg/L), no qual pode ser observado que a irradiação resulta em um aumento na área do voltamograma e diminuição do potencial da RDO, de uma forma análoga ao comportamento observada no eletrólito suporte (Figura 2a). De acordo com a literatura, a carga voltamétrica anódica $\left(\mathrm{Q} / \mathrm{mC}^{-2}\right)$ pode ser considerada uma medida aproximada da área de eletrodos de óxido ${ }^{41}$. Valores de carga obtidos a partir dos voltamogramas cíclicos são apresen- 
Tabela 1. Cargas anódicas obtidas pelos 3 corantes em meio de $\mathrm{Na}_{2} \mathrm{SO}_{4}\left(0,02 \mathrm{~mol} \mathrm{~L}^{-1}\right)$

\begin{tabular}{lcccc}
\hline Corante & \multicolumn{2}{c}{$\mathrm{Q} / \mathrm{mC} \mathrm{cm}{ }^{-2}$} & $\Delta \mathrm{Q} / \mathrm{mC} \mathrm{cm}^{-2}$ & $\begin{array}{c}\text { Com de aumento } \\
\text { de } \mathrm{Q}^{*}\end{array}$ \\
& irradiação & irradiação $\mathrm{UV}$ & $\Delta \mathrm{Q}_{\mathrm{ES}}=17,75$ & - \\
\hline A Eletrólito Suporte & 57,70 & 75,45 & $\Delta \mathrm{Q}_{\mathrm{AOR}}=17,10$ & $-3,4$ \\
B Amarelo ouro remazol RNL & 58,15 & 75,25 & $\Delta \mathrm{Q}_{\mathrm{AR}}=18,85$ & $+6,5$ \\
C Alaranjado remazol 3R & 56,98 & 75,83 & $\Delta \mathrm{Q}_{\mathrm{PR}}=19,20$ & $+8,2$ \\
D Preto remazol B & 68,15 & 87,35 & & + \\
\hline
\end{tabular}

*comparado com valor de Q obtido no eletrólito suporte durante irradiação.

tados na Tabela 1 para o eletrólito suporte e os três corantes estudados. Também são apresentados na Tabela os valores do aumento porcentual de Q na presença de irradiação, comparados com Q do eletrólito suporte.

Os valores na Tabela 1 demonstram que no caso do corante AOR ocorre uma diminuição de Q na presença de irradiação, enquanto que para PR e AR Q aumenta. Estas observações levam a concluir que a oxidação do corante $\mathrm{PR}$ pode ocorrer com mais facilidade no eletrodo de $\mathrm{Ti} / \mathrm{Ru}_{0,3} \mathrm{Ti}_{0,7} \mathrm{O}_{2}$. Considerando os comprimentos de onda nos quais ocorrem as absorbâncias máximas dos cromóforos $\left(\lambda_{\mathrm{AOR}}=410, \lambda_{\mathrm{AR}}=494\right.$ e $\left.\lambda_{\mathrm{PR}}=597 \mathrm{~nm}\right)$, quanto maior o valor de $\lambda_{\max }$, maior será o aumento de $\mathrm{Q}$, indicando a ocorrência de oxidação fotossensibilizada.

\section{Oxidações eletroquímicas e fotoeletroquímicas}

\section{Análises de ultravioleta-visível}

Na Figura 3 são apresentados os espectros de UV-vis para as diferentes soluções de corantes ao longo de $3 \mathrm{~h}$ de degradação, nos quais podem ser observadas as bandas de absorção características dos corantes, sendo a degradação indicada pelo decréscimo destas bandas.

A espectrometria no ultravioleta pode ser aplicada em sistemas conjugados, lembrando que a absorção molecular na região do

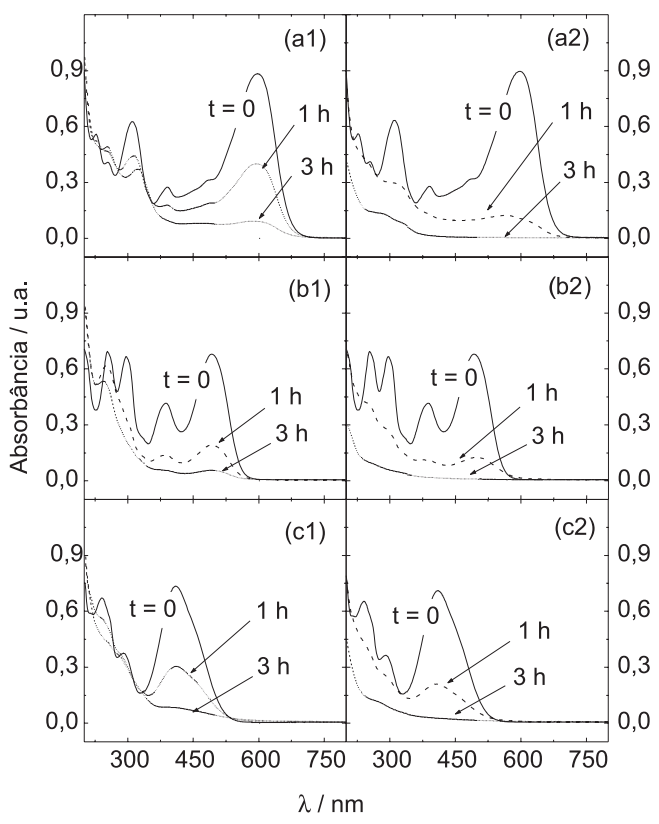

Figura 3. Espectros na região de UV-vis de (A) preto remazol B; (B) alaranjado remazol $3 R$ e $(C)$ amarelo ouro remazol $R N L$ correspondentes às degradações: (1) eletroquímica e (2) fotoeletroquímica. As curvas correspondem aos diferentes tempos de degradação, como indicado em cada figura ultravioleta e do visível é dependente de cada estrutura eletrônica molecular ${ }^{49}$. Quando há ligações duplas conjugadas, a energia necessária para uma transição é mais baixa e a absorção é deslocada para comprimentos de onda maiores, podendo assim ser mais facilmente medida. Quando existem muitas ligações duplas conjugadas a absorção é deslocada para a região do visível e é assim que um composto adquire $\operatorname{cor}^{50}$.

A razão pela qual os compostos azo são intensamente coloridos deve-se, em geral, ao fato da ligação azo (diazenodiila), -N=N-, fazer conjugação com dois anéis aromáticos. Esta conjugação fornece um sistema estendido de deslocamento de elétrons $\pi$ e permite absorção de luz na região do visível. De acordo com Pinheiro et $a l .^{51}$, os espectros de UV-vis podem ser divididos por linhas verticais denominadas zonas espectrais, com identificações aproximadas. Neste contexto, o autor dividiu os espectros em 3 zonas: zona I, entre 200 e $250 \mathrm{~nm}$, correspondente às interferências prováveis de ocorrer nos efluentes têxteis; zona II, entre 250 e 350 nm, na qual o espectro pode ser explorado para a detecção de aminas e zona III, entre 350 e $700 \mathrm{~nm}$, na qual é possível quantificar níveis de corantes residuais.

Pela observação dos espectros, são encontradas as seguintes bandas: para PR (Figura 3 a1 e a2): 597 nm (cromóforo); $389 \mathrm{~nm}$ (grupo azo); $252 \mathrm{~nm}$ (anéis benzênicos); para AR (Figura 3 b1 e b2): $494 \mathrm{~nm}$ (cromóforo); $386 \mathrm{~nm}$ (grupo azo); $295 \mathrm{~nm}$ (ácido gama acetilado); $254 \mathrm{~nm}$ (anéis benzênicos) e para AOR (Figura $3 \mathrm{c} 1 \mathrm{e}$ c2): $410 \mathrm{~nm}$ (cromóforo); 238 e 290 (grupo 3-amina-acetanilida).

A banda característica do agrupamento azo pode não ser observada isoladamente no espectro do AOR, devido ao fato que a banda correspondente à cor $(410 \mathrm{~nm})$ está muito próxima da região característica desse grupo. Na Figura 3 é observado ainda que o decréscimo da absorbância máxima das bandas é muito mais acentuado para o tratamento combinado (FEQ) que para o tratamento simples (EQ), sendo que esta tendência pode ser observada para todos os corantes estudados.

Em um trabalho recente deste laboratório foi observado que durante a eletrooxidação de ácido húmico, sobre $\mathrm{Ti} / \mathrm{Ru}_{0,3} \mathrm{Ti}_{0,7} \mathrm{O}_{2}$, a quantidade de intermediários formados é menor quando o processo é auxiliado por luz ultravioleta ${ }^{49}$. Portanto, os espectros apresentados na Figura 3 confirmam esta observação. Na região de 225 a $375 \mathrm{~nm}$, observa-se, para todos espectros, que a diminuição da banda pelo método EQ é muito aquém daquela do FEQ.

O percentual da redução da cor foi quantificado pelo acompanhamento do decréscimo relativo nas bandas que caracterizam a respectiva cor de cada corante e, assim, foram obtidos os dados apresentados na Figura 4. Pela comparação dos dados fica evidente que a maior velocidade de descoloração refere-se à aplicação do processo FEQ; entretanto, qualitativamente, observa-se que o corante PR é degradado mais rápido que os demais. Além disso, a ordem de desaparecimento de cor segue a ordem $\mathrm{PR}>\mathrm{AR}>\mathrm{AOR}$, em total acordo com os resultados de voltametria cíclica. 


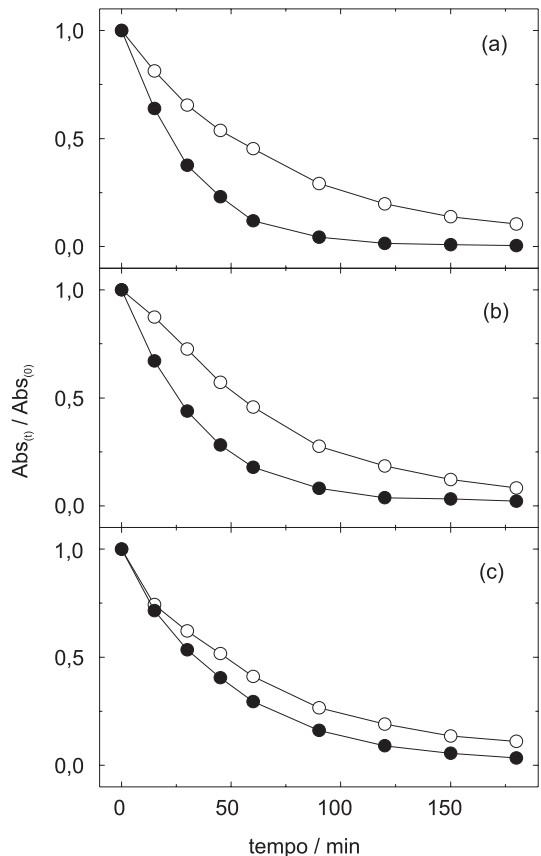

Figura 4. Grau de descoloração dos corantes durante as degradações $(\bigcirc)$ eletroquímica e (@) fotoeletroquímica; (a) preto remazol $B\left(\lambda_{\max }=597 \mathrm{~nm}\right)$; (b) alaranjado remazol $3 R\left(\lambda_{\max }=494 \mathrm{~nm}\right)$ e (c) amarelo ouro remazol $R N L$ $\left(\lambda_{\max }=410 \mathrm{~nm}\right)$

\section{Cinética da descoloração}

Diferentes estudos indicam que a oxidação de substâncias orgânicas sobre eletrodos de óxido obedece a cinética de pseudo primeira ordem, devido à formação de sítios ativos $\mathrm{MO}_{\mathrm{x}+1}$, nos quais não ocorre adsorção da espécie orgânica ${ }^{42,44,52}$. Assim, o desaparecimento da cor foi analisado no presente trabalho em termos de cinética simples de $1^{\text {a }}$ ordem, pela construção de um gráfico de $\ln \left(\mathrm{Abs}_{\mathrm{t}} / \mathrm{Abs}_{0}\right)$ em função do tempo de eletrólise (Figura 5), cujo coeficiente linear corresponde à constante de $1^{\text {a }}$ ordem aparente, $\mathrm{k}_{\text {ap. }}$. Na Tabela 2 são apresentados os valores de $\mathrm{k}_{\text {ap }}$ obtidos, os quais permitem observar que, pelo método eletroquímico, os três corantes apresentam valores de $\mathrm{k}_{\text {ap }}$ praticamente iguais (variação de somente $0,00024 \mathrm{~min}^{-1}$ ). Por outro lado, ao ser aplicado o tratamento combinado a diferença é nítida, com variação de $0,003 \mathrm{~min}^{-1}$, sendo o corante PR degradado mais rapidamente. De fato, a ordem de degradação dos corantes em questão segue a sequiência PR $(597 \mathrm{~nm})$ $>\operatorname{AR}(494 \mathrm{~nm})>\operatorname{AOR}(410 \mathrm{~nm})$, que é a mesma seqüência observada pelos resultados da voltametria. Este fato indica que os corantes sofrem degradação fotossensibilizada, aumentando assim a velocidade de degradação (Equações 9 a 14).

Além disso, é interessante notar que os valores dos coeficientes de correlação linear (R) são melhores para as eletrólises puramente eletroquímicas $(\sim 0,99)$. Para o processo combinado observa-se que $\mathrm{R}$ é menor $(\sim 0,97)$ indicando que, possivelmente, outros

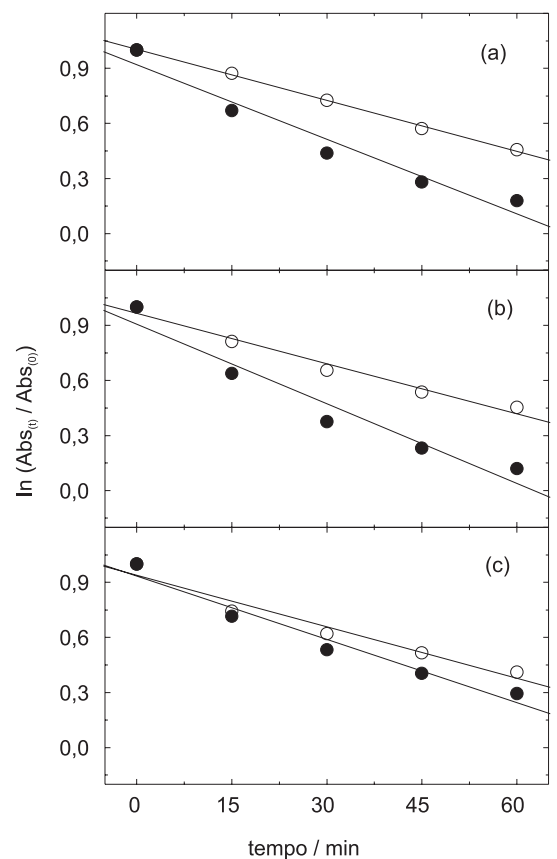

Figura 5. Curva de pseudo $1^{a}$ ordem da descoloração* com o tempo de degradação pelo processo $(\bigcirc)$ eletroquímico e $(\bullet)$ fotoeletroquímico dos corantes (a) alaranjado remazol $3 R$, (b) preto remazol B e (c) amarelo ouro remazol RNL. *Valores de absorbância foram medidos em $\lambda_{\max }$

processos ocorrem (e.g. fotossensibilização), e não somente a formação de $\mathrm{MO}_{\mathrm{X}}(\mathrm{OH})$ ou $\mathrm{MO}_{\mathrm{X}+1}$.

Assim, os valores na Tabela 2 consolidam quantitativamente a observação qualitativa das Figuras 3 a 5, onde fica claro que a velocidade da oxidação é mais rápida quando é empregado o tratamento combinado (fotoeletroquímico).

\section{Análises de Carbono Orgânico Total (COT)}

A taxa de remoção de carbono orgânico total (COT) foi determinada com o intuito de acompanhar a possível mineralização total dos corantes estudados (Tabela 2). Os resultados apresentados na Tabela 2 demonstram que o método FEQ é muito mais eficaz no processo de mineralização que o método $\mathrm{EQ}$, chegando a aumentar a quantidade removida em até $160 \%$ (para o corante AR). Pode ser observado ainda que para o corante PR ocorre a maior taxa de remoção, em ambos os métodos de tratamento.

Um fator importante em qualquer sistema de tratamento é o gasto energético associado a cada processo. Desta forma, o consumo energético $\left(\mathrm{kW} \mathrm{s}^{-1} \mathrm{~kg}^{-1}\right)$ associado com os ensaios de oxidação realizados neste trabalho foi calculado de acordo com a equação:

Consumo $=\frac{\text { U.I.t }}{\mathrm{m}}$

onde: $\mathrm{U}=$ potencial $(\mathrm{V}) ; \mathrm{I}=$ corrente $(\mathrm{A}) ; \mathrm{t}=$ tempo $(\mathrm{s})$ e $\mathrm{m}=$

Tabela 2. Potencial de operação (E), carbono orgânico total removido (COT), consumo energético e constantes de descoloração ( $\left.\mathrm{k}_{\mathrm{ap}}\right)$ de pseudo $1^{\text {a }}$ ordem para a degradação eletroquímica e fotoeletroquímica

\begin{tabular}{|c|c|c|c|c|c|c|c|c|}
\hline \multirow[t]{2}{*}{ Corantes } & \multicolumn{4}{|c|}{ Eletroquímica } & \multicolumn{4}{|c|}{ Fotoeletroquímica } \\
\hline & $\mathrm{E} / \mathrm{V}$ & $\begin{array}{c}\text { COT } \\
\text { removido/\% }\end{array}$ & $\begin{array}{l}\text { Consumo/ } \\
\text { MW s kg-1 }\end{array}$ & $\mathrm{k}_{\mathrm{ap}} / \min ^{-1}$ & $\mathrm{E} / \mathrm{V}$ & $\begin{array}{c}\text { COT } \\
\text { removido/\% }\end{array}$ & $\begin{array}{l}\text { Consumo/ } \\
\text { MW s kg-1 }\end{array}$ & $\mathrm{k}_{\mathrm{ap}} / \min ^{-1}$ \\
\hline Preto (PR) & 9,08 & 20,00 & 13,36 & 0,00912 & 8,36 & 36,40 & $6,77(50 \%)$ & 0,01445 \\
\hline Alaranjado (AR) & 9,70 & 10,50 & 22,03 & 0,00925 & 8,30 & 27,50 & $7,20(67 \%)$ & 0,01354 \\
\hline Amarelo (AOR) & 9,60 & 10,20 & 24,80 & 0,00936 & 8,44 & 23,20 & $9,58(61 \%)$ & 0,01148 \\
\hline
\end{tabular}


massa removida $(\mathrm{kg})$.

Pelos dados da Tabela 2 pode ser observado que a degradação do corante PR apresenta o menor consumo energético para ambos os processos, devido principalmente ao menor potencial de operação. Entretanto, ao ser aplicado o processo fotoeletroquímico o potencial da célula sofre uma diminuição significativa, indicando que uma menor quantidade de energia é gasta no processo fotoeletroquímico. De fato, ocorre uma diminuição entre 67 e $50 \%$ no consumo energético do processo combinado sobre o puramente eletroquímico.

\section{Adequação às leis ambientais}

Antes que efluentes coloridos venham a ser descartados, níveis de cor aceitáveis devem ser alcançados ${ }^{53}$. Um método para determinar se os níveis de cor estão em nível ambientalmente aceitável é pela comparação com absorbâncias estipuladas pelos órgãos públicos $^{53}$. De acordo com este método as absorbâncias na faixa de 400 a 650 nm são medidas e comparadas com o padrão permitido. A Figura 6 apresenta a variação da absorbância no espectro da luz UV-visível para o final dos tratamentos eletroquímicos e fotoeletroquímicos. As absorbâncias são comparadas com os padrões permi$\operatorname{tidos}^{53}$ e observa-se que, no caso do tratamento fotoeletroquímico, as absorbâncias finais encontram-se abaixo do permitido enquanto que o tratamento puramente eletroquímico não se mostra eficiente na descoloração. Este resultado mostra que o método fotoeletroquímico é capaz de atingir níveis de remoção de cor compatíveis com os padrões estabelecidos ${ }^{53}$.

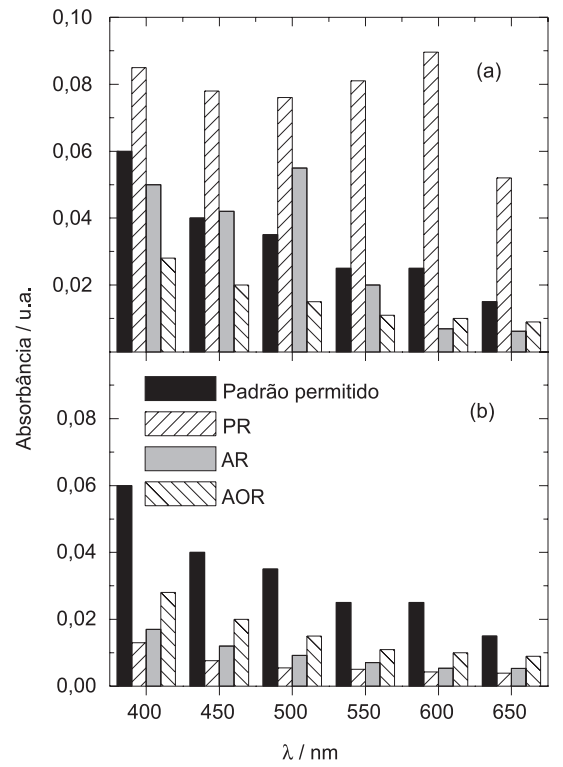

Figura 6. Variação da absorbância na região de UV-vis para os três corantes estudados, após tratamento eletroquímico (a) e fotoeletroquímico (b). As absorbâncias são comparadas com os padrões permitidos

\section{CONCLUSÕES}

Os resultados apresentados apontam para a viabilidade dos tratamentos eletroquímico e fotoeletroquímico para efluentes contendo corantes têxteis (preto remazol B, alaranjado remazol 3R e amarelo ouro remazol RNL). O método fotoeletroquímico mostrou-se mais eficiente, tanto para descoloração total ( $90 \mathrm{~min})$ quanto para a remoção de COT. Os corantes apresentando cromóforos em comprimentos de onda maiores são degradados com mais rapidez, fato este que indica que há outros processos ocorrendo - possivelmente a sensibilização do corante antes da oxidação no eletrodo. O pro- cesso fotoeletroquímico é eficiente na remoção total da cor mostrando-se assim um possível método para o pré-tratamento de efluente proveniente da indústria têxtil.

\section{AGRADECIMENTOS}

Ao apoio financeiro do CNPq e da FAPESP (04/09588-1) e também à De Nora (Brasil) por fornecer os eletrodos.

\section{REFERÊNCIAS}

1. Kunz, A.; Peralta-Zamora, P.; Gomes de Moraes, S.; Durán, N.; Quim. Nova 2002, 25, 78 .

2. Holme, I.; Developments in the chemistry and technology of organic dyes, Blackwell Scientific Publications: Oxford, 1984.

3. Booth, G.; Zollinger, H.; McLaren, K.; Sharples, W. G.; Westwall, A. Em Ullmannn's Encyclopedia of Industrial Chemistry; $5^{\text {th }}$ ed.; $\mathrm{VCH}$ : Weinheim, 1985, A9, p. 73.

4. Waring, D. R.; Hallas, G.; The Chemistry and Application of Dyes, Plenum Press: New York, 1990.

5. Gordon, P. F.; Gregory, P.; Organic Chemistry in Colour, Springer Verlag: Berlin, 1987.

6. Guaratini, C. C. I.; Zanoni, M. V. B.; Quim. Nova 2000, 23, 71.

7. Alcântara, M. R.; Daltin, D.; Quim. Nova 1996, 19, 320.

8. Nigam, P.; Banat, I. M.; Singh, D.; Marchant, R.; Process Biochem. 1996, 31,435 .

9. Sacks, J.; Buckey, C. A.; Water Sci. Technol. 1999, 40, 177.

10. Swamy, J.; Ramsay, J. A.; Enzyme Microb. Technol. 1999, 24, 130.

11. Knapp, J. S.; Newby, P. S. T.; Water Res. 1995, 29, 1807.

12. Lee, Y. H.; Pavlostathis, S. G.; Water Res. 2004, 38, 1838.

13. Karcher, S.; Kornmüller, A.; Jekel, M.; Water Sci. Technol. 1999, 40, 425.

14. Karcher, S.; Kornmuller, A.; Jekel, M.; Water Res. 2001, 35, 3317.

15. Tünay, O.; Kabdasli, I.; Eremektar, G.; Orhon, D.; Water Sci. Technol. 1996, 34,9 .

16. Mills, A.; Davies, R. H.; Worsley, D.; Chem. Soc. Rev. 1993, 22, 417.

17. Hoffman, M. R.; Martin, S. T.; Choi, W.; Bahnemann, D. W.; Chem. Rev. 1995, 95, 69.

18. Arslan, I.; Balcioglu, I. A.; Bahnemann, D. W.; Appl. Catal., B 2000, 26, 193.

19. Gouvêa, C. A. K.; Wypych, F.; Moraes, S. G.; Durán, N.; Nagata, N.; Zamora, P. P.; Chemosphere 2000, 40, 433.

20. Gonçalves, M. S. T.; Campos, A. M. F. O.; Pinto, E. M. M. S.; Plasência, P. M. S.; Queiroz, M. J. R. P.; Chemosphere 1999, 39, 781.

21. Qu, P.; Zhao, J.; Shen, T.; Hidaka, H.; J. Mol. Catal. A: Chem. 1998, 129, 257.

22. Naskar, S.; Pillay, S. A.; Chanda, M.; J. Photochem. Photobiol., A 1998, $113,257$.

23. Shourong, Z.; Qingguo, H.; Jun, Z.; Bingkun, W.; J. Photochem. Photobiol., A 1997, 108, 235.

24. Vinodgopal, K.; Wynkoop, D. E.; Kamat, P. V.; Environ. Sci. Technol. 1996, 30,1660 .

25. Konstantinou, I. K.; Albanis, T. A.; Appl. Catal., B 2004, 49, 1.

26. Wu, T.; Lin, T.; Zhao, J.; Hidaka, H.; Serpone, N.; Environ. Sci. Technol. 1999, 33, 1379.

27. Wu, T.; Liu, G.; Zhao, J.; Hidaka, H.; Serpone, N.; J. Phys. Chem. 1999, $103,4862$.

28. Vinodgopal, K.; Kamat, P. V.; Chemtech. 1996, 26, 18.

29. Tryk, D. A.; Fujishima, A.; Honda, K.; Electrochim. Acta 2000, 45, 2363.

30. Vinogopal, K.; Hotchandani, S.; Kamat, P. V.; J. Phys. Chem. 1993, 97, 9040.

31. Vinogopal, K.; Stafford, U.; Gray, K. A.; Kamat, P. V.; J. Phys. Chem. 1994, $98,6797$.

32. Vinogopal, K.; Kamat, P. V.; Sol. Energy Mater. Sol. Cells 1995, 38, 401.

33. Walder, G.; Pourmodjib, M.; Bauer, R.; Neumann-Spallart, M.; Chemosphere 2003, 50, 989.

34. Byrne, J. A.; Davidson, A.; Dunlop, P. S. M.; Eggins, B. R.; J. Photochem. Photobiol., A 2002, 148, 365.

35. Na, T.; Xiong, Y.; Li, G.; Zha, C.; Zhu, X.; J. Photochem. Photobiol., A 2002, 152, 155 .

36. Candal, R. J.; Zeltner, W. A.; Anderson, M. A.; Environ. Sci. Technol. 2000, $34,3443$.

37. Harper, J. C.; Christensen, P. A.; Egerton, T. A.; Curtis, T. P.; Gunlazuardi, J.; J. Appl. Electrochem. 2001, 31, 623.

38. Vinodgopal, K.; Kamat, P. V.; Chem. Mater. 1996, 8, 2180. 
39. Zanoni, M. V. B.; Sene, J. J.; Anderson, M. A.; Photochem. Photobiol., A $\mathbf{2 0 0 3}, 157,55$

40. Hepel, M.; Luo, J.; Electrochim. Acta 2001, 47, 729.

41. Trasatti, S.; Electrochim. Acta 1991, 36, 225.

42. Comninellis, Ch.; Electrochim. Acta 1994, 39, 1857.

43. Pelegrino, R. L.; Di Iglia, R. A.; Sanches, C. G.; Avaca, L. A.; Bertazzoli, R.; J. Braz. Chem. Soc. 2002, 13, 60.

44. Malpass, G. R. P.; Motheo A. J.; Appl. Electrochem. 2001, 31, 1351.

45. Pelegrini, R.; Peralta-Zamora, P.; de Andrade, A. R.; Reyes, J.; Duran, N.; Appl. Catal., B 1999, 22, 83.

46. Pelegrini, R.; Bertazzoli, R.; Quim. Nova 2002, 25, 477.

47. Pelegrini, R. T.; Freire, R. S.; Duran, N.; Bertazzoli, R.; Environ. Sci. Technol. 2001, 35, 2849.
48. Catanho, M.; Malpass, G. R. P.; Motheo, A. J.; Appl. Catal., B 2006, 62, 193.

49. Pinhedo, L.; Pelegrini, R.; Bertazzoli, R.; Motheo, A. J.; Appl. Catal., B 2004, 57, 81 .

50. Silverstein, R. M.; Bassler, G. C.; Morril, T. C.; Identificação Espetrométrica de Compostos Orgânicos, Guanabara Koogan S.A. Ed.: Rio de Janeiro, 1994

51. Pinheiro, H. M.; Touraud, E.; Thomas, O.; Dyes Pigm. 2004, 61, 121

52. Malpass, G. R. P.; Motheo A. J.; J. Braz. Chem. Soc. 2003, 14, 65.

53. Cooper, P.; J. Soc. Dyers and Colour 1993, 97, 98. 